H. Saito

Nagoya Math. J.

Vol. 187 (2007), 49-73

\title{
EVEN-RELATIVE-DIMENSIONAL VANISHING CYCLES IN BIVARIANT INTERSECTION THEORY
}

\author{
HIROSHI SAITO
}

\begin{abstract}
For a smooth variety proper over a curve having a fibre with isolated ordinary quadratic singularities, it is well-known that we have the vanishing cycles associated to the singularities in the étale cohomology of the geometric generic fibre. The base-change by a double cover of the base curve ramified at the image of the singular fibre has singularities corresponding to the singularities in the fibre. In this note, we show that in the even relative-dimensional case, there exist elements of the bivariant Chow group of the base-change with supports in the singularities and hence their images in the bivariant Chow group with supports in the special fibre and that the usual cohomological vanishing cycles are obtained as their images by a natural map, a kind of "cycle map" so that the elements in the bivariant Chow groups can be regarded as the vanishing cycles. The bivariant Chow group with supports in the special fibre has a ring structure and the natural map is a ring homomorphism to the cohomology ring of the geometric generic fibre. Also discussed is the relation of the bivariant Chow group with supports in the special fibre to the specialization map of Chow groups.
\end{abstract}

The vanishing cycles are studied extensively since Picard in the study of topology of algebraic varieties and live in (co-)homology groups. In this note, we shall show that the vanishing cycles of isolated ordinary quadratic singularities of even relative-dimensional case live in appropriate bivariant Chow groups, and from which the usual vanishing cycles are obtained by natural maps.

In order to state our result more precisely, we review the Chow bivariant theory briefly $([\mathrm{F}], 17)$. We denote the (usual) Chow group of an algebraic scheme $X$ by $C H .(X)$. For a morphism $f: X \rightarrow Y$ of algebraic schemes, an element of $C H^{p}(X \stackrel{f}{\rightarrow} Y)$ is a collection of maps $c=\left\{c_{g}: C H .\left(Y^{\prime}\right) \rightarrow C H .{ }_{-p}\left(X \times_{Y} Y^{\prime}\right)\right\}$ for all morphisms $g: Y^{\prime} \rightarrow Y$ satisfying certain compatibility conditions with proper push-forward, flat

Received April 30, 1998.

Revised December 19, 2003, May 16, 2005, September 27, 2005, March 28, 2006, July 18, 2006.

2000 Mathematics Subject Classification: 14C17, 14F17. 
pull-backs and pull-back by regular embeddings. In the sequel, we always put $X^{\prime}=X \times_{Y} Y^{\prime}$. The image $c_{g}\left(\left[Y^{\prime}\right]\right) \in C H_{m-p}\left(X^{\prime}\right)$ is called $c$ evaluated at $\left[Y^{\prime}\right]$ if $\operatorname{dim} Y^{\prime}=m$. In particular, for a base field $k$, we have a bijection $C H^{-q}(X \rightarrow \operatorname{Spec} k) \cong C H_{q}(X)$ by evaluation at [Spec $\left.k\right]$. The bivariant theory has three operations:

(1) We have the pull-back $g^{*}(c) \in C H^{p}\left(X^{\prime} \rightarrow Y^{\prime}\right)$ for $c \in C H^{p}(X \rightarrow Y)$ and for any morphism $g: Y^{\prime} \rightarrow Y$ by restriction.

(2) For $X \stackrel{f}{\rightarrow} Y \stackrel{g}{\rightarrow} Z$ and $c \in C H^{p}(X \rightarrow Y)$ and $b \in C H^{q}(Y \rightarrow Z)$, we have $c \cdot b \in C H^{p+q}(X \rightarrow Z)$, the multiplication defined by composition.

(3) For $X \stackrel{f}{\rightarrow} Y \stackrel{g}{\rightarrow} Z$ with $f$ proper and $d \in C H^{p}(X \rightarrow Z)$, we have the proper push-forward $f_{*}(d) \in C H^{p}(Y \rightarrow Z)$.

These operations have some compatibility conditions including the projection formula. For a flat morphism $g: Y \rightarrow Z$ of pure relative dimension $d$, the flat pull-back defines an element $[g] \in C H^{-d}(Y \rightarrow Z)$ called the orientation class. If $g: Y \rightarrow Z$ is smooth, the composition $\cdot[g]: C H^{p}(X \rightarrow$ $Y) \rightarrow C H^{p-d}(X \rightarrow Z)$ is bijective. In particular, if $Y$ is smooth of pure dimension $d$, then $C H^{p}(X \rightarrow Y) \cong C H^{p-d}(X \rightarrow \operatorname{Spec}(k)) \cong C H_{d-p}(X)$. For a regular embedding $g: Y \rightarrow Z$ of codimension $d$, the pull-back by $g$ defines also an orientation class $[g] \in C H^{d}(Y \rightarrow Z)$. It is known that a local complete intersection morphism, i.e. a regular embedding followed by a smooth morphism defines an orientation class, as a composite of corresponding orientation classes, which is independent of the decomposition ([F], 6.6). We define $C H^{p}(X)=C H^{p}\left(X \stackrel{\mathrm{id}_{X}}{\longrightarrow} X\right)$. This is isomorphic to the Chow group of $X$ of dimension $\operatorname{dim} X-p$ for $X$ smooth, but it is not in general. Notice that $C H^{\cdot}(X)$ has a ring structure by composition.

Let $f^{\prime}: Y \rightarrow T$ be a morphism from smooth algebraic variety of dimension $2 r+1$ to a smooth curve over algebraically closed field of characteristic $\neq 2$ such that the fibre $Y_{s^{\prime}}$ over a point $s^{\prime} \in T$ has only isolated ordinary quadratic singularities $W$ and let $\varphi: S \rightarrow T$ be a double covering ramified at $s \in S$ with $\varphi(s)=s^{\prime}$ and $f: X \rightarrow S$ be the base-change of $f^{\prime}$ and $V=W \times_{T}\{s\} \subset X$. We choose a (strict) henselization $s \rightarrow S^{h} \rightarrow S$. We shall denote the étale cohomology of a scheme $X$ with $\mathbf{Z}_{\ell^{-c o e f f i c i e n t}}$ $(\ell \neq$ char. $k)$ by $H^{\cdot}(X)$. Then, we have

TheOREM. For each point $v \in V$, we have

$$
C H^{r+1}(v \rightarrow X) \cong \mathbf{Z} .
$$


Let $\Delta_{v} \in C H^{r+1}\left(X_{s} \rightarrow X\right)$ be the image of a generator by the map $C H^{r+1}(v \rightarrow X) \rightarrow C H^{r+1}\left(X_{s} \rightarrow X\right)$ induced by the natural embedding $v \rightarrow X_{s}$ and $X_{\bar{t}}$ be the geometric generic fibre of $X \rightarrow S$. Then there exists a natural map $C H^{r+1}\left(X_{s} \rightarrow X\right) \rightarrow H^{2 r}\left(X_{\bar{t}}\right)$ sending $\Delta_{v} \in C H^{r+1}\left(X_{s} \rightarrow X\right)$ to the vanishing cycle $\delta_{v} \in H^{2 r}\left(X_{\bar{t}}\right)$ with respect to the point $v$.

Thus, the vanishing cycle $\Delta_{v}$ lives in the bivariant group $C H^{r+1}\left(X_{s} \rightarrow\right.$ $X)$ and the cohomological vanishing cycle $\delta_{v}$ is its image by the natural map.

The map $C H^{r+1}\left(X_{s} \rightarrow X\right) \rightarrow H^{2 r}\left(X_{\bar{t}}\right)$ appearing in the theorem is in fact a part of collection of maps

$$
C H^{p+1}\left(X_{s} \rightarrow X\right) \longrightarrow H^{2 p}\left(X_{\bar{t}}\right)
$$

for $0 \leq p \leq 2 r$. They have the following properties.

The collection $C H^{+1}\left(X_{s} \rightarrow X\right)$ has a ring structure such that the map

$$
C H^{\cdot+1}\left(X_{s} \rightarrow X\right) \longrightarrow H^{2 \cdot}\left(X_{\bar{t}}\right)
$$

is a ring homomorphism.

The diagram

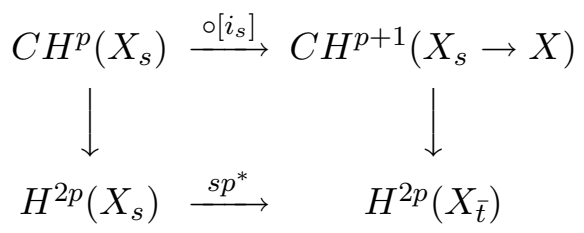

is commutative, where the left vertical map is the "natural" cycle map and $i_{s}: X_{s} \rightarrow X$ is the embedding and $s p^{*}: H^{2 p}\left(X_{s}\right) \rightarrow H^{2 p}\left(X_{\bar{t}}\right)$ is the specialization map.

If $p$ and $q$ are integers with $p+q=2 r$, we have the specialization map

$$
s p_{*}: C H_{q}\left(X_{\bar{t}}\right) \longrightarrow C H_{q}\left(X_{s}\right)
$$

of Chow groups (cf. [F] 20.3 and 1.12 below), which factors as

$$
s p_{*}: C H_{q}\left(X_{\bar{t}}\right) \stackrel{\sigma}{\longrightarrow} C H^{p+1}\left(X_{s} \rightarrow X\right) \longrightarrow C H_{q}\left(X_{s}\right)
$$

where the second map is the evaluation at $[X]$. Then the composite

$$
C H^{p}\left(X_{\bar{t}}\right) \stackrel{\sigma}{\longrightarrow} C H^{p+1}\left(X_{s} \rightarrow X\right) \longrightarrow H^{2 p}\left(X_{\bar{t}}\right)
$$


is the cycle map. Moreover the vanishing cycle $\Delta_{v}$ vanishes in $C H_{q}\left(X_{s}\right)$.

It is worthwhile to note that we do not assume the vanishing cycle algebraic. Hence a transcendental cycle can be represented in terms of bivariant Chow theory.

As an application we give a formula for the intersection number of the vanishing cycle with an algebraic cycle in terms of the tangent cone at the singular point (cf. 1.16).

\section{$\S 1$. Vanishing cycles in bivariant groups}

Recall that a morphism of algebraic schemes is said to be an envelope if it is proper and every fibre over a schematic point has a rational point. Recall also that the envelopes define a Grothendieck topology.

Theorem 1.1. (Kimura $[\mathrm{K}]$ ) For a morphism $X \rightarrow Y$ of schemes, the functor $Y^{\prime} \mapsto C H^{p}\left(X \times_{Y} Y^{\prime} \rightarrow Y^{\prime}\right)$ is a sheaf for the Grothendieck topology of envelopes of $Y$.

1.2. Let $f: X \rightarrow S$ be a morphism of schemes of dimension $2 r+1$ $(r>0)$ to a smooth curve over an algebraically closed field $k$ of characteristic $\neq 2, s \in S$ a point. We assume that the morphism $f$ is smooth over $S \backslash\{s\}$, and that the fibre $X_{s}$ has only isolated ordinary quadratic singularities and they are also isolated ordinary quadratic singularities of $X$. We denote the set of singular points of $X$ by $V$. Recall that a point of a variety is an isolated ordinary (or non-degenerate in our case) quadratic singularity if it is formally or étale-locally isomorphic to the origin of

$$
q=\sum_{i, j=1}^{n+1} a_{i j} x_{i} x_{j}=0
$$

in the affine space with $a_{i j} \in k$ and $\operatorname{det}\left(a_{i j}\right) \neq 0$. Here instead of assuming $\operatorname{det}\left(a_{i j}\right) \neq 0$, we may assume that $\left(a_{i j}\right)$ is the identity matrix; or that $q=$ $x_{1} x_{2}+\cdots+x_{2 m-1} x_{2 m}$ if $n=2 m-1$ and $q=x_{1} x_{2}+\cdots+x_{2 m-1} x_{2 m}+x_{2 m+1}^{2}$ if $n=2 m$. Let $\pi: \tilde{X} \rightarrow X$ be the blowing-up along the singular points of $X$, $Q$ be the exceptional divisor. Then $\tilde{X}$ is smooth and $Q=\coprod_{v \in V} Q_{v}$ and each $Q_{v}$ is a smooth quadric in $\mathbf{P}^{2 r+1}$. Moreover, let $\hat{X}_{s}$ be the blow-up of the fibre $X_{s}$ along $V$ and, $Q^{\prime}=Q \cap \hat{X}_{s}$ be the exceptional divisor of $\hat{X}_{s}$. Then $\hat{X}_{s}$ is smooth and the strict transform of the fibre $X_{s}$ and $Q^{\prime}=\coprod_{v \in V} Q_{v}^{\prime}$ and each $Q_{v}^{\prime}$ is a smooth quadric in $\mathbf{P}^{2 r}$. 
Proposition 1.3. Let $Y$ be a closed subscheme of $X$, and

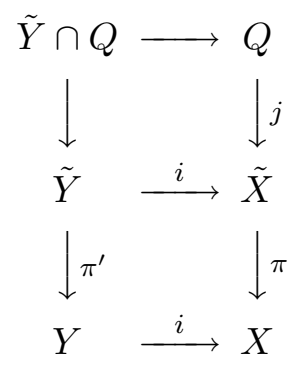

be Cartesian squares. Then for each integer $p$, if $p \neq 0$ or $Y \neq X$, we have

$$
C H^{p}(Y \rightarrow X) \cong \operatorname{Ker}\left(j^{!}: C H_{2 r+1-p}(\tilde{Y}) \rightarrow C H_{2 r-p}(\tilde{Y} \cap Q)\right) .
$$

Proof. Note that

$$
X^{\prime}=\tilde{X} \times_{X} \tilde{X}=\tilde{X} \cup\left(Q \times_{X} Q\right) .
$$

Putting $Y^{\prime}=X^{\prime} \times_{X} Y$, we have the exact sequence

$$
0 \longrightarrow C H^{p}(Y \rightarrow X) \longrightarrow C H^{p}(\tilde{Y} \rightarrow \tilde{X}) \longrightarrow C H^{p}\left(Y^{\prime} \rightarrow X^{\prime}\right)
$$

by the above theorem, where the second map is the difference of the maps induced by the first and second projections from $X^{\prime}$ to $\tilde{X}$. Since $\tilde{X} \amalg\left(Q \times_{X}\right.$ $Q) \rightarrow X^{\prime}$ is an envelope, $C H^{p}\left(Y^{\prime} \rightarrow X^{\prime}\right)$ injects into $C H^{p}(\tilde{Y} \rightarrow \tilde{X}) \oplus$ $C H^{p}\left(Y \times_{X} Q \times_{X} Q \rightarrow Q \times_{X} Q\right)$. Hence, we have an exact sequence

$0 \longrightarrow C H^{p}(Y \rightarrow X) \longrightarrow C H^{p}(\tilde{Y} \rightarrow \tilde{X}) \longrightarrow C H^{p}\left(Y \times{ }_{X} Q \times{ }_{X} Q \rightarrow Q \times{ }_{X} Q\right)$.

The second map factors as

$$
C H^{p}(\tilde{Y} \rightarrow \tilde{X}) \longrightarrow C H^{p}\left(Y \times{ }_{X} Q \rightarrow Q\right) \longrightarrow C H^{p}\left(Y \times_{X} Q \times_{X} Q \rightarrow Q \times{ }_{X} Q\right)
$$

and the latter map, the difference of the maps induced by the first and the second projections $\left.p_{1}, p_{2}: Q \times_{X} Q \rightarrow Q\right)$ is injective for $p>0$ : the problem is local on $X$ by 1.1 , we may assume the singular point is unique. Then choosing a point $q$ on $Q$, we have injections

$$
i_{1}, i_{2}: Q \longrightarrow Q \times_{X} Q
$$

defined by $i_{1}(x)=(x, q)$, and $i_{2}(x)=(q, x)$. Assume for $x \in C H^{p}\left(Y \times_{X}\right.$ $Q \rightarrow Q)$, we have $p_{1}^{*}(x)-p_{2}^{*}(x)=0$. Notice that $p_{1} \circ i_{1}=i d_{Q}$ and $p_{2} \circ i_{1}$ 
factors through a point. Applying $i_{1}^{*}$ to the equality, we get $x-i_{1}^{*} \circ p_{2}^{*}(x)=$ $x-\left(p 2 \circ i_{1}\right)^{*}(x)=0$. The element $\left(p_{2} \circ i_{1}\right)^{*}(x)$ is the image of an element of $C H^{p}\left(Y \times_{X} q \rightarrow q\right) \cong C H_{-p}\left(Y \times_{X} q\right)$, which vanishes unless $p \leq 0$. We conclude the injectivity for $p>0$. Thus, we have the exact sequence

$$
0 \longrightarrow C H^{p}(Y \rightarrow X) \longrightarrow C H^{p}(\tilde{Y} \rightarrow \tilde{X}) \longrightarrow C H^{p}(Q \cap \tilde{Y} \rightarrow Q) .
$$

Since $\tilde{X}$ and $Q$ are smooth, we have the natural identifications

$$
\begin{aligned}
C H^{p}(\tilde{Y} \rightarrow \tilde{X}) & =C H_{2 r+1-p}(\tilde{Y}), \\
C H^{p}(Q \cap \tilde{Y} \rightarrow Q) & =C H_{2 r-p}(Q \cap \tilde{Y}),
\end{aligned}
$$

and we can identify the map $C H^{p}(\tilde{Y} \rightarrow \tilde{X}) \rightarrow C H^{p}(Q \cap \tilde{Y} \rightarrow Q)$ with $j^{!}: C H_{2 r+1-p}(\tilde{Y}) \rightarrow C H_{2 r-p}(\tilde{Y} \cap Q)$. For $p=0$, we have

$$
C H^{0}(\tilde{Y} \rightarrow \tilde{X}) \cong C H_{2 r+1}(\tilde{Y})=0
$$

unless $Y=X$ by dimension reason, and clearly, $C H^{0}(X \rightarrow X)=\mathbf{Z}$.

Corollary 1.4. If $v$ is a singular point of $X$, we have

$$
C H^{p}(v \rightarrow X)= \begin{cases}\mathbf{Z} & \text { if } p=r+1 \\ 0 & \text { otherwise }\end{cases}
$$

Proof. By Proposition 1.3, we have

$$
C H^{p}(v \rightarrow X)=\operatorname{Ker}\left(j^{!}: C H_{2 r+1-p}\left(Q_{v}\right) \rightarrow C H_{2 r-p}\left(Q_{v}\right)\right)
$$

where $Q_{v}$ denotes the exceptional divisor lying over the point $v$. By the excess intersection formula ([F], Theorem 6.3), we have $j^{!}=c_{1}\left(N_{Q_{v} / \tilde{X}}\right) \cup$ $: C H_{2 r+1-p}\left(Q_{v}\right) \rightarrow C H_{2 r-p}\left(Q_{v}\right)$. Since $N_{Q_{v} / \tilde{X}}=\mathcal{O}_{Q_{v}}\left(Q_{v}\right)=-h_{v}$, where $h_{v}$ is the hyperplane section of $Q_{v}$, the above proposition implies the desired formula (cf. 1.17).

Corollary 1.5. We have exact sequences

$$
0 \longrightarrow C H^{p}\left(X_{s} \rightarrow X\right) \longrightarrow C H_{2 r+1-p}\left(\tilde{X}_{s}\right) \longrightarrow C H_{2 r-p}(Q) .
$$

The following proposition follows similarly from Theorem 1.1. 
Proposition 1.6. The sequence

$$
0 \longrightarrow C H^{p}\left(\tilde{X}_{s}\right) \longrightarrow C H^{p}\left(\hat{X}_{s}\right) \oplus C H^{p}(Q) \longrightarrow C H^{p}\left(Q^{\prime}\right)
$$

is exact.

Proposition 1.7. There is an exact sequence

$$
C H_{q}\left(Q^{\prime}\right) \stackrel{-}{\longrightarrow} C H_{q}\left(\hat{X}_{s}\right) \oplus C H_{q}(Q) \stackrel{+}{\longrightarrow} C H_{q}\left(\tilde{X}_{s}\right) \longrightarrow 0 .
$$

Proof. Consider the fibre square

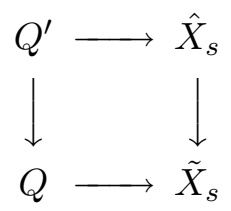

and notice $\tilde{X}_{s} \backslash Q=\hat{X}_{s} \backslash Q^{\prime}$. We have the desired exact sequence by [F], Example 1.8.1, where the first arrow is the difference of push-forwards induced by the natural inclusions and the second map is the sum of push-forwards induced by the natural inclusions.

Proposition 1.8. We have an exact sequence

$$
0 \longrightarrow C H_{2 r-p}\left(Q^{\prime}\right) \longrightarrow C H^{p}\left(\tilde{X}_{s}\right) \longrightarrow C H^{p+1}\left(X_{s} \rightarrow X\right) \longrightarrow 0
$$

Proof. Indeed, we have a diagram whose horizontal sequences are exact:

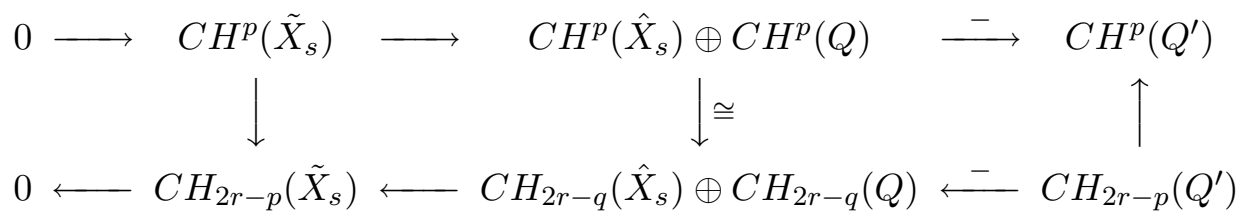

We shall show that the right vertical arrow is zero; then we have an injection $0 \rightarrow C H_{2 r-p}\left(Q^{\prime}\right) \rightarrow C H^{p}\left(\tilde{X}_{s}\right)$ and its cokernel injects into $C H_{2 r-p}\left(\tilde{X}_{s}\right)$. Notice that the map $C H^{p}\left(\hat{X}_{s}\right) \oplus C H^{p}(Q) \rightarrow C H^{p}\left(Q^{\prime}\right)$ is identified with

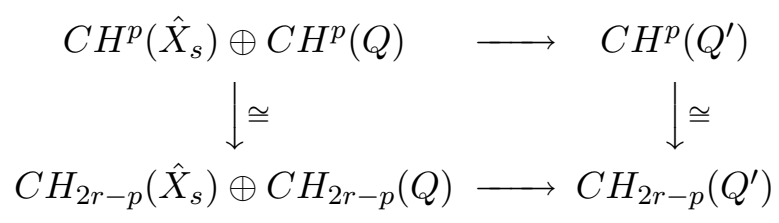


where the lower map is the difference of the maps induced by the injections $i: Q^{\prime} \hookrightarrow Q, j: Q^{\prime} \hookrightarrow \hat{X}_{s}$. Hence, the image of $z \in C H_{2 r-p}\left(Q^{\prime}\right)$ in $C H_{2 r-1-p}\left(Q^{\prime}\right)$ is $i^{!} i_{*}(z)+j^{!} j_{*}(z)$. By the excess intersection formula, we have

$$
\begin{aligned}
i^{!} i_{*}(z)+j^{!} j_{*}(z) & =c_{1}\left(N_{Q^{\prime} / Q}\right) \cup z+c_{1}\left(N_{Q^{\prime} / \hat{X}_{s}}\right) \cup z \\
& =\left(c_{1}\left(N_{Q^{\prime} / Q}\right)+c_{1}\left(N_{Q^{\prime} / \hat{X}_{s}}\right) \cup z ;\right.
\end{aligned}
$$

but since $c_{1}\left(N_{Q^{\prime} / Q}\right)$ is the hyperplane section of quadric, while $c_{1}\left(N_{Q^{\prime} / \hat{X}_{s}}\right)$ is the minus of the hyperplane section of quadric, it vanishes. We thus have an exact sequence

$$
0 \longrightarrow C H_{2 r-p}\left(Q^{\prime}\right) \longrightarrow C H^{p}\left(\tilde{X}_{s}\right) \longrightarrow C H_{2 r-p}\left(\tilde{X}_{s}\right) .
$$

To determine the image, consider the diagrams

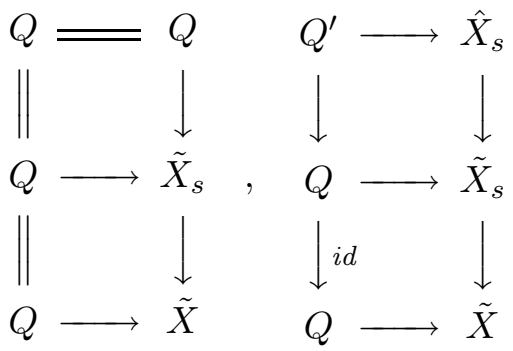

and we see that the following diagram commutes, where the lower map is induced by the inclusion $Q \hookrightarrow \tilde{X}$ :

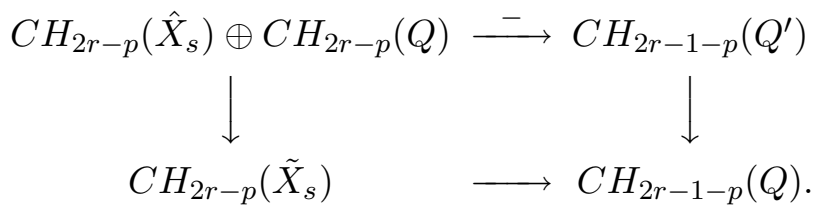

Since the right vertical arrow is injective, the image in $\mathrm{CH}_{2 r-p}\left(\tilde{X}_{s}\right)$ of the kernel of the horizontal arrow above, i.e., the image of $C H^{p}\left(\tilde{X}_{s}\right)$, is the kernel of the horizontal arrow below, that is, $C H^{p+1}\left(X_{s} \rightarrow X\right)$.

Proposition 1.9. The ring structure of $C H \cdot\left(\tilde{X}_{s}\right)$ induces the ring structure on $C H^{+1}\left(X_{s} \rightarrow X\right)$ from the map $C H^{p}\left(\tilde{X}_{s}\right) \rightarrow C H^{p+1}\left(X_{s} \rightarrow X\right)$. 
Proof. It suffices to show that the multiplication of an element of $C H^{\cdot}\left(\tilde{X}_{s}\right)$ by an element of $C H\left(Q^{\prime}\right)$ is in $C H\left(Q^{\prime}\right)$. Note that the map

$$
C H^{\cdot}\left(\tilde{X}_{s}\right) \longrightarrow C H\left(\hat{X}_{s}\right) \oplus C H(Q)=C H\left(\hat{X}_{s}\right) \times C H(Q)
$$

is a ring homomorphism and that $\hat{X}_{s}$ and $Q$ are smooth, so we can and we do identify the Chow cohomology and the Chow homology. Let $i$ and $j$ denote the inclusions $Q^{\prime} \hookrightarrow Q$ and $Q^{\prime} \hookrightarrow \hat{X}_{s}$. For $z \in C H\left(\tilde{X}_{s}\right)$, its image in $C H\left(\hat{X}_{s}\right) \times C H(Q)$ is of the form $(x, y), x \in C H\left(\hat{X}_{s}\right), y \in C H(Q)$ with $i^{*}(y)=j^{*}(x)=w$. The image of $C H\left(Q^{\prime}\right)$ is of the form $\left(j_{*}(u),-i_{*}(u)\right)$, $u \in C H\left(Q^{\prime}\right)$. Then, we get

$$
\begin{aligned}
(x, y) \cdot\left(j_{*}(u),-i_{*}(u)\right) & =\left(x \cdot j_{*}(u),-y \cdot i_{*}(u)\right) \\
& =\left(j_{*}\left(j^{*}(x) \cdot u\right),-i_{*}\left(i^{*}(y) \cdot u\right)\right) \\
& =\left(j_{*}(w \cdot u),-i_{*}(w \cdot u)\right)
\end{aligned}
$$

so the product is in the image of $C H\left(Q^{\prime}\right)$.

Remark 1.10. For $p \neq 2 r, C H_{2 r-p}\left(Q^{\prime}\right) \hookrightarrow C H^{p}\left(\tilde{X}_{s}\right)$ can be identified with $C H^{p}\left(Q^{\prime} \rightarrow \tilde{X}_{s}\right) \hookrightarrow C H^{p}\left(\tilde{X}_{s}\right)$, but not for $p=2 r$.

DEFinition 1.11. We denote a generator of the kernel of multiplication by the hyperplane section of the Chow group of codimension $r$ of $2 r$ dimensional quadric by $\Delta$. For a singular point $v$ of $X$, there is a cycle $\Delta_{v} \in C H^{r}\left(\tilde{X}_{s}\right)$ whose restriction to $Q_{v}$ is the cycle $\Delta$ and whose restriction to the other $Q_{v^{\prime}}$ and to $\hat{X}_{s}$ vanish, by 1.6. We denote its image in $C H^{r+1}\left(X_{s} \rightarrow X\right)$ also by $\Delta_{v}(1.8)$. It is the image by the map induced by $v \rightarrow X_{s}$ of a generator of $C H^{r+1}(v \rightarrow X)$, and hence is determined up to sign.

When $X$ is proper over $S$, we have the degree map

$$
\langle\rangle: C H^{2 r+1}\left(X_{s} \rightarrow X\right) \longrightarrow C H_{0}\left(X_{s}\right) \longrightarrow \mathbf{Z}
$$

defined by

$$
\langle c\rangle=\int_{X_{s}} c([X])
$$

for $c \in C H^{2 r+1}\left(X_{s} \rightarrow X\right)$. If $c_{1}+c_{2} \in C H^{2 r}\left(\hat{X}_{s}\right) \oplus C H^{2 r}(Q) \cong C H^{2 r}\left(\tilde{X}_{s}\right)$ is a lift of $c(1.6)$, then

$$
\langle c\rangle=\int_{\hat{X}_{s}} c_{1}\left(\left[\hat{X}_{s}\right]\right)+\int_{Q} c_{2}([Q])
$$


which is independent of the choice of the lifting, by definition of the map $C H\left(Q^{\prime}\right) \rightarrow C H\left(\hat{X}_{s}\right) \oplus C H(Q)$ and the isomorphisms $C H_{0}\left(\hat{X}_{s}\right) \cong C H_{0}\left(X_{s}\right)$, $C H_{0}\left(Q^{\prime}\right) \cong C H_{0}(Q)$. Note that we have (cf. 1.17 below)

$$
\left\langle\Delta_{v}^{2}\right\rangle=(-1)^{r} 2
$$

Theorem 1.12. Fix a (strict) henselization $s \rightarrow S^{h}$ of $s \rightarrow S$ and let $X_{\bar{t}}$ be the geometric generic fibre of $X \rightarrow S$ and $p, q$ be integers with $p+q=2 r$. We have the specialization map sp $p_{*}: C H_{q}\left(X_{\bar{t}}\right) \rightarrow C H_{q}\left(X_{s}\right)$. There is a map $\sigma: C H^{p}\left(X_{\bar{t}}\right) \rightarrow C H^{p+1}\left(X_{s} \rightarrow X\right)$ such that

$$
s p_{*}: C H_{q}\left(X_{\bar{t}}\right) \stackrel{\sigma}{\longrightarrow} C H^{p+1}\left(X_{s} \rightarrow X\right) \longrightarrow C H_{q}\left(X_{s}\right)
$$

where the second map is the evaluation at $[X]$.

Proof. Recall the definition of the specialization map (cf. [F] 20.3). For a finite extension $L$ of the function field $\kappa(t)$ in the field $\kappa(\bar{t})$, let $S^{\prime \prime}$ be the normalization of $S^{h}$ in $L \cdot \kappa\left(t^{h}\right)$, where $t^{h}$ is the generic point of the henselization $S^{h}$. Recall that a field $k$ is a Nagata (= universally-japanese [EGA], chapt $0,23.1 .1$ ) ring [B], chap. $\mathrm{V}, \S 3$, no 2 , théorème 2 , hence so is the local ring $\mathcal{O}_{S, s}$, therefore its henselization ${ }^{h} \mathcal{O}_{S, s}$ is also a Nagata ring [EGA], IV, 18.7.3. Because $L \cdot \kappa\left(t^{h}\right)$ is a finite extension of $\kappa\left(t^{h}\right), S^{\prime \prime}$ is finite over $S^{h}$. Since $S^{h}$ is henselian and $S^{\prime \prime}$ is integral hence connected, $S^{\prime \prime}$ is a local ring with residue field $k$. Thus, $s \rightarrow S^{h}$ factors uniquely as $s \rightarrow S^{\prime \prime} \rightarrow S^{h}$. Let $S^{\prime}$ be the normalization of $S$ in $L$, we have a map $S^{\prime \prime} \rightarrow S^{\prime}$, hence, a pointed (smooth) curve $s \rightarrow S^{\prime} \rightarrow S$. If $L_{1}$ is a finite extension of $L$, and if $s \rightarrow S_{1}^{\prime}$ is the corresponding pointed curve, we have a map of pointed curves $s \rightarrow S_{1}^{\prime} \rightarrow S^{\prime}$. We consider the system of (germs of) pointed curves thus obtained. Let $s \rightarrow S^{\prime} \rightarrow S$ be the pointed (smooth) curve smooth over $S$ outside $s$. Consider the base-change

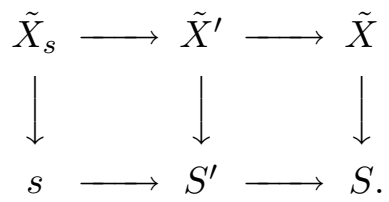

Then we get the natural isomorphism $\tilde{X} \backslash \tilde{X}_{s} \cong X \backslash X_{s}$. Denote the inclusion $s \rightarrow S^{\prime}$ by $k^{\prime}$. We have the exact sequence

$$
C H_{q+1}\left(\tilde{X}_{s}\right) \stackrel{k_{*}^{\prime}}{\longrightarrow} C H_{q+1}\left(\tilde{X}^{\prime}\right) \longrightarrow C H_{q+1}\left(\tilde{X}^{\prime} \backslash \tilde{X}_{s}\right) \longrightarrow 0
$$


and the map $k^{\prime !}: C H_{q+1}\left(\tilde{X}^{\prime}\right) \rightarrow C H_{q}\left(\tilde{X}_{s}\right)$. Since $k^{\prime !} \circ k_{*}^{\prime}=0$ holds by the excess intersection formula, and because of the fact that the normal bundle $N_{\{s\} / S^{\prime}}$ on the point $\{s\}$ is trivial, we get a map

$$
C H_{q+1}\left(\tilde{X}^{\prime} \backslash \tilde{X}_{s}\right) \longrightarrow C H_{q}\left(\tilde{X}_{s}\right) .
$$

Taking the limit of these maps for various $S^{\prime}$, we get the desired specialization map

$$
C H_{q}\left(X_{\bar{t}}\right) \longrightarrow C H_{q}\left(\tilde{X}_{s}\right)
$$

For the inclusion $i: Q \rightarrow \tilde{X}$, we claim that $0=i^{!} \circ k^{\prime !}: C H_{q+1}\left(\tilde{X}^{\prime}\right) \rightarrow$ $C H_{q-1}(Q)$. Look at the diagram

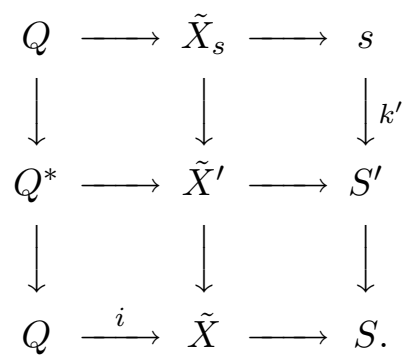

Then we get $i^{!} \circ k^{\prime !}=k^{\prime !} \circ i^{!}$by $[\mathrm{F}], 6.4$. Since $s \in S^{\prime}$ is the only point over $s \in S$, and the image of $Q$ in $S$ is $s, Q^{*}{ }_{r e d}=Q$. Hence $C H(Q) \stackrel{\cong}{\rightarrow} C H\left(Q^{*}\right)$. From the diagram

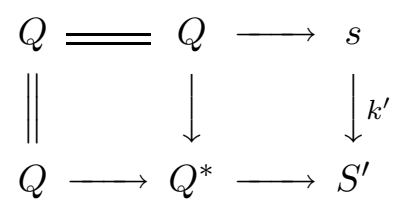

and the excess intersection formula, we conclude $0=k^{\prime !}: C H_{q}\left(Q^{*}\right) \rightarrow$ $C H_{q-1}(Q)$. This means that the image of $k^{\prime !}: C H_{q+1}\left(\tilde{X}^{\prime}\right) \rightarrow C H_{q}\left(\tilde{X}_{s}\right)$ is in the kernel of $i^{!}: C H_{q}\left(\tilde{X}_{s}\right) \rightarrow C H_{q-1}(Q)$, that is, in $C H^{p+1}\left(X_{s} \rightarrow X\right)$ by (1.5). Therefore we have $\sigma: C H^{p}\left(X_{\bar{t}}\right) \rightarrow C H^{p+1}\left(X_{s} \rightarrow X\right)$ and the specialization map factors as

$$
\sigma: C H^{p}\left(X_{\bar{t}}\right) \stackrel{\sigma}{\longrightarrow} C H^{p+1}\left(X_{s} \rightarrow X\right) \longrightarrow C H_{q}\left(\tilde{X}_{s}\right) .
$$

Since the specialization maps commute with the push-forwards, the diagram

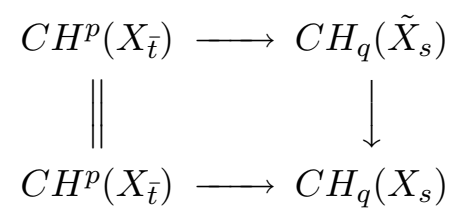


commutes, where the horizontal arrows are specializations and the right vertical arrow is push-forward, from which the theorem follows.

Remark 1.13. We have an exact sequence

$$
0 \longrightarrow \bigoplus_{v} C H^{r+1}(v \rightarrow X) \longrightarrow C H^{r+1}\left(X_{s} \rightarrow X\right) \longrightarrow C H_{r}\left(X_{s}\right) \longrightarrow 0 .
$$

In fact, it follows from Propositions 1.3 and 1.7 that we have the exact sequence, since $C H_{r}(Q) \rightarrow C H_{r-1}(Q)$ is surjective.

Proposition 1.14. The maps $\sigma: C H^{p}\left(X_{\bar{t}}\right) \rightarrow C H^{p+1}\left(X_{s} \rightarrow X\right)$ form a ring homomorphism.

Proof. Let $s \rightarrow S^{\prime} \rightarrow S$ be as in the previous proof. We shall show that the map $C H^{p}\left(\tilde{X}^{\prime}\right) \rightarrow C H^{p}\left(\tilde{X}^{\prime} \backslash \tilde{X}_{s}\right) \cong C H_{q+1}\left(\tilde{X}^{\prime} \backslash \tilde{X}_{s}\right)$ is surjective. We have the diagram

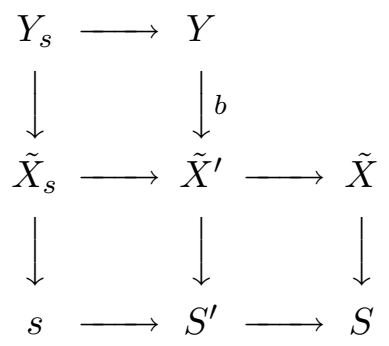

where $b: Y \rightarrow \tilde{X}^{\prime}$ is a resolution of singularities of $\tilde{X}^{\prime}$. (See 2.7 below for the resolution.) We may assume that $Y \backslash Y_{s} \cong \tilde{X}^{\prime} \backslash \tilde{X}_{s}$. Note that the map

$$
C H^{p}(Y) \cong C H_{q+1}(Y) \longrightarrow C H_{q+1}\left(Y \backslash Y_{s}\right)=C H^{p}\left(Y \backslash Y_{s}\right)
$$

is surjective. Since the diagram

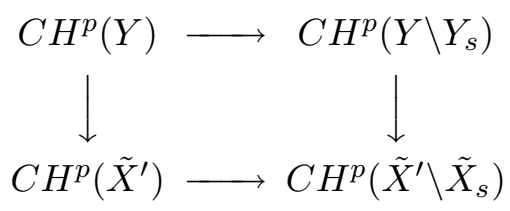

commutes, where the left vertical arrow is given by $x \mapsto b_{*}(x \circ[b])$, and the right vertical arrow is bijective, the restriction map

$$
C H^{p}\left(\tilde{X}^{\prime}\right) \longrightarrow C H^{p}\left(\tilde{X}^{\prime} \backslash \tilde{X}_{s}\right)
$$


is surjective and this map factors as

$$
C H^{p}\left(\tilde{X}^{\prime}\right) \longrightarrow C H_{q+1}\left(\tilde{X}^{\prime}\right) \longrightarrow C H^{p}\left(\tilde{X}^{\prime} \backslash \tilde{X}_{s}\right)=C H_{q+1}\left(\tilde{X}^{\prime} \backslash \tilde{X}_{s}\right) .
$$

The diagram

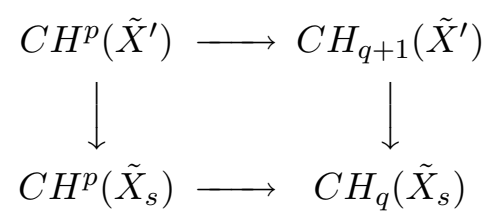

commutes, where the upper horizontal map is evaluation at $\left[\tilde{X}^{\prime}\right]$, the lower horizontal map is evaluation at $\left[\tilde{X}_{s}\right]$, and the vertical maps are the restrictions. Therefore, we get the commutative diagram

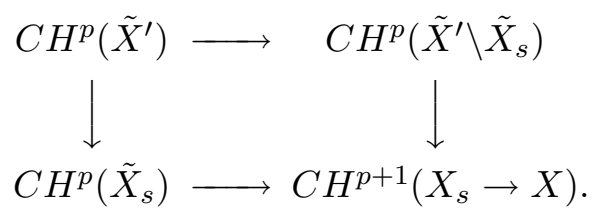

The upper horizontal map is surjective and the maps except the right vertical arrow form ring homomorphisms, hence so do the right vertical ones. By passing to the limit, we see that the induced map $C H^{\cdot}\left(\tilde{X}_{\bar{t}}\right) \rightarrow C H^{\cdot+1}\left(X_{s} \rightarrow\right.$ $X)$ is a ring homomorphism.

Proposition 1.15. Let $s \rightarrow S^{\prime}$ be a pointed curve as in the proof of the theorem (hence $s \rightarrow S^{\prime}$ is the unique point over $s \rightarrow S$ ) with the ramification index $e$ of the morphism $S^{\prime} \rightarrow S$ at $s$ and consider the cartesian square

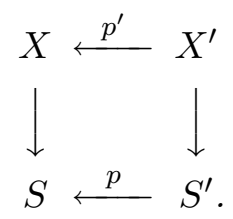

Let $Z^{\prime} \subset X^{\prime}$ be an integral subscheme of dimension $r+1$ and $C_{v^{*}} Z^{\prime}$ its normal cone along the intersection $v^{*}=p^{\prime-1}(v) \cap Z^{\prime}$ of $p^{\prime-1}(v)$ and $Z^{\prime}$. We have inclusions

$$
C_{v^{*}} Z^{\prime} \subset C_{p^{\prime-1}(v) / X^{\prime}}=C_{v} X \times_{s} p^{-1}(s) \subset T_{v} X \times_{s} p^{-1}(s)
$$

and their projective bundles $P\left(C_{v^{*}} Z^{\prime}\right) \subset P\left(C_{v} X\right) \times_{s} p^{-1}(s) \subset P\left(T_{v} X\right) \times_{s}$ $p^{-1}(s)$. Notice that $P\left(C_{v} X\right) \subset P\left(T_{v} X\right)$ can be naturally identified with the 
inclusion $Q \subset \mathbf{P}^{2 r+1}$. Under these assumptions and notations, we can write uniquely

$$
\left[P\left(C_{v^{*}} Z^{\prime}\right)\right]=e \cdot Z^{*} \quad \text { as a cycle on } P\left(C_{v^{*}} Z^{\prime}\right) \subset Q
$$

and the image of geometric generic fibre $Z_{\bar{t}}^{\prime}$ of $Z$ over $S$ by the map $C H^{r}\left(X_{\bar{t}}\right) \rightarrow C H^{r+1}\left(X_{s} \rightarrow X\right) \hookrightarrow C H_{r}\left(\tilde{X}_{s}\right)$ is $Z^{*}+\left(\right.$ a cycle on $\left.\hat{X}_{s}\right)$ and the product of $\Delta_{v}$ and the image $\sigma\left(Z_{\bar{t}}^{\prime}\right)$ in $C H^{\cdot}\left(X_{s} \rightarrow X\right)$ is given by $Z^{*} \cdot \Delta_{v}$ in $Q$.

Proof. We consider $S^{\prime}$ Zariski-locally around $s \rightarrow S^{\prime}$. Let $I_{v} \subset \mathcal{O}_{X}$ be the ideal sheaf of the point $v$. Then the blow-up $\tilde{X}$ is, by definition,

$$
\tilde{X}=\operatorname{Proj}\left(\bigoplus_{n \geq 0} I_{v}^{n}\right) \text {. }
$$

Note that $p$, hence $p^{\prime}$ is flat. Let $I_{Z^{\prime}} \subset \mathcal{O}_{X^{\prime}}$ be the ideal sheaf of $Z^{\prime}$, and put $J_{v}=\left(p^{\prime *}\left(I_{v}\right)+I_{Z^{\prime}}\right) / I_{Z^{\prime}} \subset \mathcal{O}_{Z^{\prime}}$. Notice that $J_{v}$ is the ideal sheaf of $v^{*}$ in $Z^{\prime}$. The strict transform of $Z^{\prime}$ in $\tilde{X}^{\prime}=\operatorname{Proj}\left(\bigoplus_{n \geq 0} p^{* *}\left(I_{v}^{n}\right)\right)$ is

$$
\tilde{Z}^{\prime}=\operatorname{Proj}\left(\bigoplus_{n \geq 0} J_{v}^{n}\right)
$$

The pull-back $Q^{*}$ of the exceptional divisor $Q$ by the morphism $\tilde{X}^{\prime} \rightarrow \tilde{X}$ is $e \cdot Q$, where the latter $Q$ is considered as a divisor lying on $\tilde{X}_{s} \subset \tilde{X}^{\prime}$. The pull-back of $\tilde{Z}^{\prime}$ by the inclusion $Q^{*} \subset \tilde{X}^{\prime}$ is given by

$$
\operatorname{Proj}\left(\bigoplus_{n \geq 0}\left(\mathcal{O}_{X^{\prime}} / p^{\prime *}\left(I_{v}\right)\right) \otimes J_{v}^{n}\right)=\operatorname{Proj}\left(\bigoplus_{n \geq 0}\left(J_{v}^{n} / J_{v}^{n+1}\right)\right)
$$

which is nothing but the projective bundle associated with the normal cone $C_{v^{*}} Z^{\prime}$ and

$$
Q=\operatorname{Proj}\left(\bigoplus_{n \geq 0}\left(I_{v}^{n} / I_{v}^{n+1}\right)\right)
$$

is the projective bundle associated with the normal cone $C_{v} X$. Since $Q^{*}$. $\left[\tilde{Z}^{\prime}\right]=e Q \cdot\left[\tilde{Z}^{\prime}\right]$ on $Q^{*} \cap \tilde{Z}^{\prime}=P\left(C_{v^{*}} Z^{\prime}\right)$, the unique existence of $Z^{*}=Q \cdot\left[\tilde{Z}^{\prime}\right]$ is trivial. We have $\tilde{X}_{s}=Q+\hat{X}_{s}$ as divisors on $\tilde{X}^{\prime}$, hence $\tilde{X}_{s} \cdot\left[\tilde{Z}^{\prime}\right]=$ $Q \cdot\left[\tilde{Z}^{\prime}\right]+\hat{X}_{s} \cdot\left[\tilde{Z}^{\prime}\right]=Z^{*}+\hat{X}_{s} \cdot\left[\tilde{Z}^{\prime}\right]$. The proposition follows from the definitions of the maps and products. 
Now we get the following corollary.

Corollary 1.16. Let $Z \subset X$ be a subvariety and $Z_{\bar{t}}$ be the geometric generic fibre. Then the intersection number $\left\langle\sigma\left(Z_{\bar{t}}\right) \cdot \Delta_{v}\right\rangle$ is $\left\langle\left[P\left(C_{v} Z\right)\right] \cdot \Delta\right\rangle$ where $P\left(C_{v} Z\right) \subset P\left(C_{v} X\right)=Q$. In particular, if $Z$ is a smooth subvariety of $X$ of dimension $r+1$ passing through the point $v$, then $\left\langle\sigma\left(Z_{\bar{t}}\right) \cdot \Delta_{v}\right\rangle= \pm 1$. For another such a variety $Z^{\prime},\left\langle\sigma\left(Z_{\bar{t}}\right) \cdot \Delta_{v}\right\rangle=\left\langle\sigma\left(Z_{\bar{t}}^{\prime}\right) \cdot \Delta_{v}\right\rangle$ if the parities of $r$ and $\operatorname{dim}\left(T_{v} Z \cap T_{v} Z^{\prime}\right)$ are opposite and $\left\langle\sigma\left(Z_{\bar{t}}\right) \cdot \Delta_{v}\right\rangle=-\left\langle\sigma\left(Z_{\bar{t}}^{\prime}\right) \cdot \Delta_{v}\right\rangle$, otherwise.

Proof. Everything is clear except the last one. It follows from the next proposition.

Proposition 1.17. Let $Q$ be a smooth quadric in $\mathbf{P}^{m+1}$ and $h \in$ $C H^{1}(Q)$ be the hyperplane section of $Q$. Then the Chow groups of $Q$ are as follows:

Case $m=2 r-1 . C H^{p}(Q)=\mathbf{Z} h^{p}$ for $0 \leq p<r$ and $C H^{p}(Q)=\mathbf{Z} h^{p} / 2$ for $r \leq p \leq m$.

Case $m=2 r . C H^{p}(Q)=\mathbf{Z} h^{p}$ for $0 \leq p<r$ and $C H^{p}(Q)=\mathbf{Z} h^{p} / 2$ for $r<p \leq m$. For $p=r, C H^{r}(Q)=\mathbf{Z}\left[L_{1}\right] \oplus \mathbf{Z}\left[L_{2}\right]$, where $L_{1}, L_{2} \subset Q$ are $r$-dimensional planes.

The ring structure of $C H^{\cdot}(Q)$ is given by $\left[L_{i}\right] \cdot h^{j}=h^{r+j} / 2$ for $i=1,2$ and $0<j \leq r$. We have

$$
\left\langle[L] \cdot\left[L^{\prime}\right]\right\rangle= \begin{cases}1 & \text { if } \operatorname{dim} L \cap L^{\prime} \text { is even } \\ 0 & \text { if } \operatorname{dim} L \cap L^{\prime} \text { is odd }\end{cases}
$$

for $r$-dimensional planes $L, L^{\prime} \subset Q$. Here we adopt the convention $\operatorname{dim} \emptyset=$ -1 .

Except the last part on the intersection numbers, the proposition is well-known. For the last part, note that the rational equivalence and the algebraic equivalence coincide on a smooth quadrics and see $[\mathrm{H}]$, XIII, 4, Theorem III.

EXAMPLE 1.18. If $Z^{\prime} \subset X^{\prime}$ is smooth at $v^{\prime}$, then the intersection number $\left\langle\sigma\left(\left[Z_{\bar{t}}^{\prime}\right]\right) \cdot \Delta_{v}\right\rangle$ is \pm 1 . 
First we shall show that $Z^{\prime} \rightarrow S^{\prime}$ is smooth at $v^{\prime}$. In fact, assume that the differential map $T_{v^{\prime}} Z^{\prime} \rightarrow T_{S^{\prime}} S^{\prime}$ vanishes. Since $T_{v^{\prime}} Z^{\prime}=C_{v^{\prime}} Z^{\prime} \subset$ $C_{v^{\prime}} X^{\prime} \subset T_{v^{\prime}} X^{\prime}$, we have

$$
T_{v^{\prime}} Z^{\prime} \subset C_{v^{\prime}} X^{\prime} \cap \operatorname{Ker}\left(T_{v^{\prime}} X^{\prime} \rightarrow T_{s^{\prime}} S^{\prime}\right)
$$

The kernel $\operatorname{Ker}\left(T_{v^{\prime}} X^{\prime} \rightarrow T_{s^{\prime}} S^{\prime}\right)$ is $(2 r+1)$-dimensional and $C_{v^{\prime}} X^{\prime} \subset T_{v^{\prime}} X^{\prime}$ is a quadric, the linear space $T_{v^{\prime}} Z^{\prime}$ of dimension $r+1$ is in zero locus of a quadratic form of $(2 r+1)$-dimensional space. Since $X_{s^{\prime}}^{\prime}$ has an isolated ordinary quadratic singularity at $v^{\prime}$, the quadratic form is nondegenerate, which is impossible. We can choose the coordinates $x_{0}, \ldots, x_{2 r+1}$ at $v^{\prime}$ (or lifts of a basis of the Zariski tangent space), so that $x_{0}$ is a local parameter of $S^{\prime}$ at $s^{\prime}$, and that $x_{0}, \ldots, x_{r}$ are the local coordinates of $Z^{\prime}$ at $v^{\prime}$. Then with the notation in the proof of Proposition 1.15, $J_{v^{*}}=\left(x_{0}{ }^{e}, x_{1}, \ldots, x_{2 r+1}\right)$ and $C_{v^{*}} Z^{\prime}=\operatorname{Spec}\left(k\left[x_{0}\right] /\left(x_{0}{ }^{e}\right)\right) \times T_{v^{\prime}} Z^{\prime}$. Hence, $P\left(C_{v^{*}} Z^{\prime}\right)=e P\left(T_{v^{\prime}} Z^{\prime}\right)$ as a cycle, and $Z^{*}=P\left(T_{v^{\prime}} Z^{\prime}\right) \subset P\left(C_{v} X\right)$; we get the intersection number as is stated above.

\section{§2. Comparison with cohomology}

2.1. We fix a (strict) henselization $s \rightarrow S^{h}$ of $s \rightarrow S$ and assume that the morphism $f: X \rightarrow S$ is proper. We denote the étale cohomology of $Y$ with coefficients in $\Lambda=\mathbf{Z}_{\ell}$ by $H^{n}(Y)$, where $\ell$ is a prime invertible in $k$ (for simplicity of notation, we omit the Tate twist). Standard reference for the formalism of the vanishing cycle in the sequel is [SGA 7], exposé XIII and exposé XV. We have

Proposition 2.2. We have a canonical map $c l_{\tilde{X}_{s}}: C H^{p}\left(\tilde{X}_{s}\right) \rightarrow$ $H^{2 p}\left(\tilde{X}_{s}\right)$ which forms a ring homomorphism. Similarly, we have a canonical map $c_{X_{s}}: C H^{p}\left(X_{s}\right) \rightarrow H^{2 p}\left(X_{s}\right)$ which forms a ring homomorphism.

Proof. We have the exact sequence

$$
0 \longrightarrow C H^{p}\left(\tilde{X}_{s}\right) \longrightarrow C H^{p}\left(\hat{X}_{s}\right) \oplus C H^{p}(Q) \stackrel{-}{\longrightarrow} C H^{p}\left(Q^{\prime}\right)
$$

and similarly, by the Mayer-Vietoris sequence, we get the exact sequence

$$
0 \longrightarrow H^{2 p}\left(\tilde{X}_{s}\right) \longrightarrow H^{2 p}\left(\hat{X}_{s}\right) \oplus H^{2 p}(Q) \stackrel{-}{\longrightarrow} H^{2 p}\left(Q^{\prime}\right)
$$


Since $\hat{X}_{s}, Q$ and $Q^{\prime}$ are smooth, we have the commutative diagram

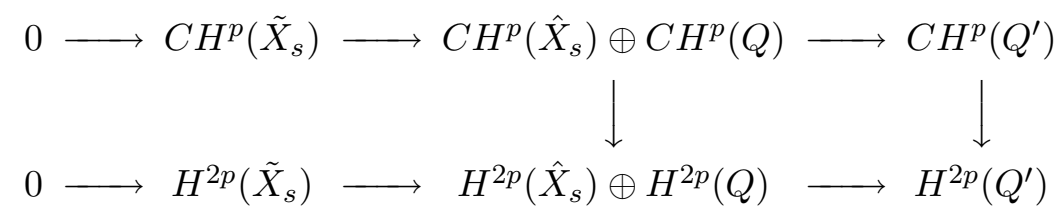

and we get the map $c l_{\tilde{X}_{s}}: C H^{p}\left(\tilde{X}_{s}\right) \rightarrow H^{2 p}\left(\tilde{X}_{s}\right)$ making the left square commutative. Since the maps in the diagram form ring homomorphisms, we see that $c l_{\tilde{X}_{s}}: C H^{\cdot}\left(\tilde{X}_{s}\right) \rightarrow H^{2 \cdot}\left(\tilde{X}_{s}\right)$ is also a ring homomorphism. Similar arguments show the existence of the map $c l_{X_{s}}: C H^{p}\left(X_{s}\right) \rightarrow H^{2 p}\left(X_{s}\right)$ defined from the usual cycle map $C H^{p}\left(\hat{X}_{s}\right) \rightarrow H^{2 p}\left(\hat{X}_{s}\right)$ by considering the envelope $\hat{\pi}_{s}: \hat{X}_{s} \rightarrow X_{s}$ and the Leray spectral sequence $H^{p}\left(X_{s}, R^{q} \hat{\pi}_{s *} \Lambda\right) \Rightarrow$ $H^{p+q}\left(\hat{X}_{s}\right)$. Notice that $R^{q} \hat{\pi}_{s *} \Lambda(q>0)$ are $H^{q}\left(Q^{\prime}\right)$ supported at the point $v$ and that $H^{q}\left(Q^{\prime}\right)=0$ for odd $q$.

THEOREM 2.3. Let $s p^{*}: H^{2 p}\left(X_{s}\right) \rightarrow H^{2 p}\left(X_{\bar{t}}\right)$ be the specialization map. There exists a map

$$
C H^{p+1}\left(X_{s} \rightarrow X\right) \longrightarrow H^{2 p}\left(X_{\bar{t}}\right)
$$

making the following diagram commutative

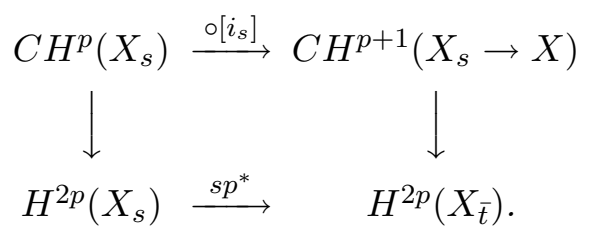

where $i_{s}: X_{s} \rightarrow X$ denotes the embedding. Moreover the map $C H^{++1}\left(X_{s} \rightarrow\right.$ $X) \rightarrow H^{2 \cdot}\left(X_{\bar{t}}\right)$ is a ring homomorphism and the image of $\Delta_{v} \in$ $C H^{p+1}\left(X_{s} \rightarrow X\right)$ is the vanishing cycle $\delta_{v} \in H^{2 r}\left(X_{\bar{t}}\right)$ relative to the point $v$, and the composite

$$
C H^{p}\left(X_{\bar{t}}\right) \stackrel{\sigma}{\longrightarrow} C H^{p+1}\left(X_{s} \rightarrow X\right) \longrightarrow H^{2 p}\left(X_{\bar{t}}\right)
$$

is the cycle map.

In fact, we prove the following. 
LEMMA 2.4. (i) Let $s p^{*}: H^{2 p}\left(\tilde{X}_{s}\right) \rightarrow H^{2 p}\left(X_{\bar{t}}\right)$ be the specialization map for $\tilde{X} \rightarrow S$. Then the kernel of the composite $C H^{p}\left(\tilde{X}_{s}\right) \rightarrow H^{2 p}\left(\tilde{X}_{s}\right) \rightarrow$ $H^{2 p}\left(X_{\bar{t}}\right)$ contains $\mathrm{CH}_{2 r-p}\left(Q^{\prime}\right)$.

(ii) Consider the map $C H^{p+1}\left(X_{s} \rightarrow X\right) \rightarrow H^{2 p}\left(X_{\bar{t}}\right)$ making the following diagram commutative

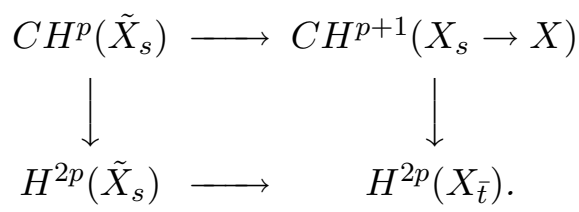

Then this map $C H^{\cdot+1}\left(X_{s} \rightarrow X\right) \rightarrow H^{2 \cdot}\left(X_{\bar{t}}\right)$ is a ring homomorphism.

Proof. To simplify the notation, we denote the base-change $X \times{ }_{S} S^{h} \rightarrow$ $S^{h}$ by $X \rightarrow S$. Without fear of confusion, we may and do identify the group $C H^{p+1}\left(X_{s} \rightarrow X\right)$ with the cokernel of $C H_{2 r-p}\left(Q^{\prime}\right) \rightarrow C H^{p}\left(\tilde{X}_{s}\right)$ by 1.8. By construction, we have a natural map $H_{4 r-2 p}\left(Q^{\prime}\right) \cong H^{2 p-2}\left(Q^{\prime}\right) \rightarrow H^{2 p}\left(\tilde{X}_{s}\right)$, which makes the following diagram commutative:

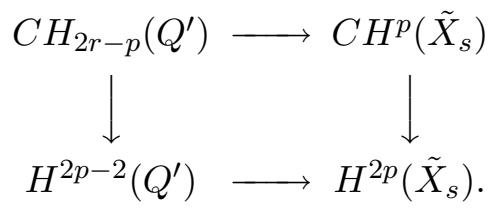

Consider the injective ring homomorphism $H^{2 p}\left(\tilde{X}_{s}\right) \rightarrow H^{2 p}\left(\hat{X}_{s}\right) \oplus H^{2 p}(Q)$. An element of $H^{2 p}\left(\tilde{X}_{s}\right)$ is identified with $(x, y) \in H^{2 p}\left(\hat{X}_{s}\right) \oplus H^{2 p}(Q)$ with $j^{*}(x)=i^{*}(y)$. For $u \in H^{2 p-2}\left(Q^{\prime}\right)$, its image is $\left(j_{*}(u),-i_{*}(u)\right)$. Let $q=2 r-p$ and $z^{\prime}=\left(x^{\prime}, y^{\prime}\right) \in H^{2 q}\left(\hat{X}_{s}\right) \oplus H^{2 q}(Q)$ with $j^{*}\left(x^{\prime}\right)=i^{*}\left(y^{\prime}\right)=w^{\prime}$. Then, the product of $u$ and $z^{\prime}$ is given by $u \cdot z^{\prime}=\left(j_{*}\left(u \cdot w^{\prime}\right),-i_{*}\left(u \cdot w^{\prime}\right)\right) \in$ $H^{4 r}\left(X_{s}\right) \oplus H^{4 r}(Q)$, which is the image of an element of $H^{4 r-2}\left(Q^{\prime}\right)$. Identifying $H^{4 r}\left(X_{\bar{t}}\right) \cong$, we have

$$
\begin{aligned}
s p^{*}\left(u \cdot z^{\prime}\right) & =\left\langle j_{*}\left(u \cdot w^{\prime}\right)\right\rangle-\left\langle i_{*}\left(u \cdot w^{\prime}\right)\right\rangle \\
& =\left\langle u \cdot w^{\prime}\right\rangle-\left\langle u \cdot w^{\prime}\right\rangle \\
& =0
\end{aligned}
$$

where the brackets denote the intersection numbers. Now let us show that the maps $s p^{*}: H^{2 p}\left(\tilde{X}_{s}\right) \rightarrow H^{2 p}\left(X_{\bar{t}}\right)$ are surjective when tensored with $\mathbf{Q}$. 
The proof will be completed in Subsection 2.6. Before embarking in the proof, notice that, since the intersection pairing

$$
H^{2 p}\left(X_{\bar{t}}\right)_{\mathbf{Q}} \times H^{2 q}\left(X_{\bar{t}}\right)_{\mathbf{Q}} \longrightarrow \Lambda_{\mathbf{Q}}
$$

is perfect, we see that $s p^{*}\left(H^{2 p-2}\left(Q^{\prime}\right)\right)$ are torsion. For the proof, we need a lemma.

Lemma 2.5. We denote the image of $\Delta_{v} \in C H^{r}\left(\tilde{X}_{s}\right)$ by the map $C H^{r}\left(\tilde{X}_{s}\right) \rightarrow H^{2 r}\left(\tilde{X}_{s}, \Lambda\right)$ also by $\Delta_{v}$. Then its image by $s p^{*}: H^{2 r}\left(\tilde{X}_{s}\right) \rightarrow$ $H^{2 r}\left(X_{\bar{t}}\right)$ is the vanishing cycle $\delta_{v}$ with respect to the point $v$.

Proof. Let $\pi: Y=Q \coprod \hat{X}_{s} \rightarrow \tilde{X}_{s}$ be the natural map. Recall $Q^{\prime}=$ $Q \cap \hat{X}_{s}$. We have an exact sequence

$$
0 \longrightarrow \Lambda_{\tilde{X}_{s}} \longrightarrow \pi_{*}\left(\Lambda_{Y}\right) \longrightarrow \Lambda_{Q^{\prime}} \longrightarrow 0
$$

from which we get

$$
H_{Q}^{2 r-1}\left(\tilde{X}_{s}, \Lambda_{Q^{\prime}}\right) \longrightarrow H_{Q}^{2 r}\left(\tilde{X}_{s}, \Lambda_{\tilde{X}_{s}}\right) \longrightarrow H_{Q}^{2 r}\left(\tilde{X}_{s}, R \pi_{*}\left(\Lambda_{Y}\right)\right) \longrightarrow H_{Q}^{2 r}\left(\tilde{X}_{s}, \Lambda_{Q^{\prime}}\right)
$$

Since $H_{Q}^{n}\left(\tilde{X}_{s}, \Lambda_{Q^{\prime}}\right)=H^{n}\left(Q^{\prime}, \Lambda\right)$ and $H_{Q}^{2 r}\left(\tilde{X}_{s}, R \pi_{*}\left(\Lambda_{\tilde{X}_{s}}\right)\right)=H^{2 r}(Q, \Lambda) \oplus$ $H_{Q^{\prime}}^{2 r}\left(\hat{X}_{s}, \Lambda\right)$, we see that the sequence

$$
0 \longrightarrow H_{Q}^{2 r}\left(\tilde{X}_{s}, \Lambda\right) \longrightarrow H^{2 r}(Q, \Lambda) \oplus H_{Q^{\prime}}^{2 r}\left(\hat{X}_{s}, \Lambda\right) \stackrel{-}{\longrightarrow} H^{2 r}\left(Q^{\prime}, \Lambda\right)
$$

is exact. Moreover, we have natural maps

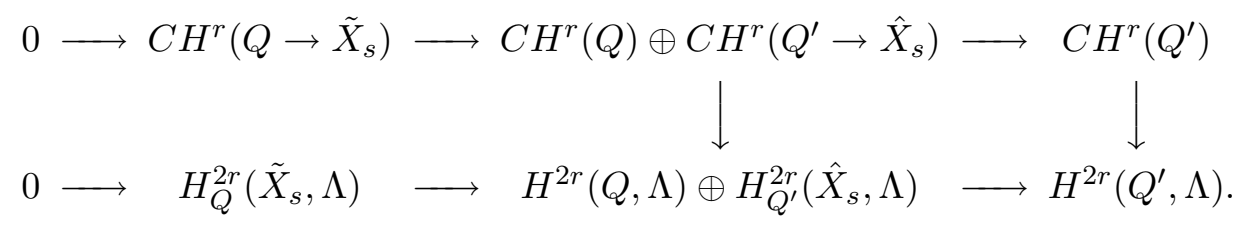

Hence we get the map

$$
C H^{r}\left(Q \rightarrow \tilde{X}_{s}\right) \longrightarrow H_{Q}^{2 r}\left(\tilde{X}_{s}, \Lambda\right)
$$

making the left square of the diagram commutative. It is clear that we have also maps $C H^{r}\left(Q_{v} \rightarrow \tilde{X}_{s}\right) \rightarrow H_{Q_{v}}^{2 r}\left(\tilde{X}_{s}, \Lambda\right)$ for each $v$ and the map above is identified with the direct sum of these maps. Note that $\Delta_{v} \in C H^{r}\left(\tilde{X}_{s}\right)$ is in the image of $C H^{r}\left(Q_{v} \rightarrow \tilde{X}_{s}\right) \rightarrow C H^{r}\left(\tilde{X}_{s}\right)$. Choose a singular point $v$ 
and let $X^{*}$ be the blowing-up of $X$ at the singular points of $X$ except $v$. We consider the diagram

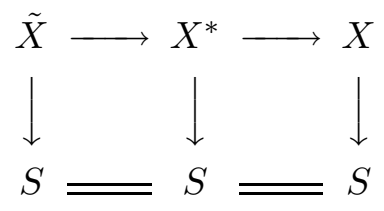

and the corresponding near-by functors $R \tilde{\psi}, R \psi^{*}, R \psi$. Recall that $H_{v}^{2 r}\left(X_{s}, R \psi \Lambda\right) \cong \Lambda$ and the vanishing cycle $\delta_{v}$ is its generator (or its image in $\left.H^{2 r}\left(X_{\bar{t}}, \Lambda\right)\right)$. We have then a commutative diagram

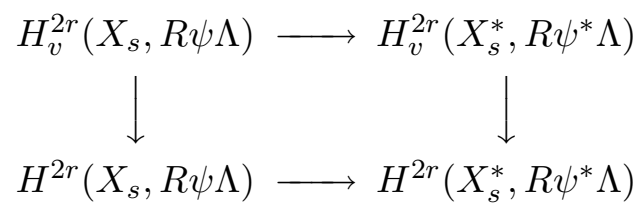

and the lower groups are isomorphic to $H^{2 r}\left(X_{\bar{t}}, \Lambda\right)$. In the diagram

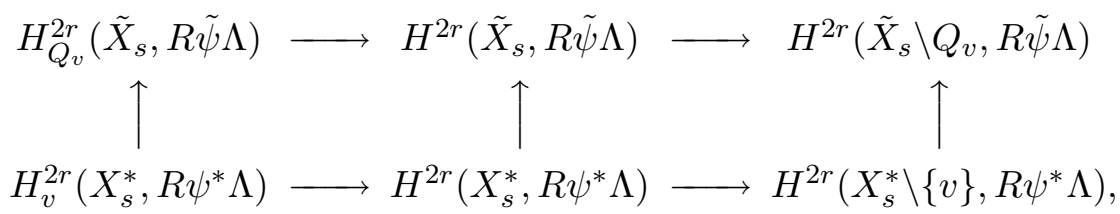

the horizontal rows are exact. The middle terms are both isomorphic to $H^{2 r}\left(X_{\bar{t}}, \Lambda\right)$. The right vertical map is isomorphic since $\tilde{X}_{s} \backslash Q_{v} \cong X^{*} \backslash\{v\}$ and the nearby functor is local on $X_{s}^{*}$, and $\tilde{X}_{s}$. Moreover, the lower left map is injective and its image is generated by the vanishing cycle $\delta_{v}$. We have a canonical map $\Lambda \rightarrow R \tilde{\psi} \Lambda$ and the image of the composite

$$
H_{Q_{v}}^{2 r}\left(\tilde{X}_{s}, \Lambda\right) \longrightarrow H_{Q_{v}}^{2 r}\left(\tilde{X}_{s}, R \tilde{\psi} \Lambda\right) \longrightarrow H^{2 r}\left(\tilde{X}_{s}, R \tilde{\psi} \Lambda\right)
$$

is contained in the image of $H_{v}^{2 r}\left(X_{s}^{*}, R \psi^{*} \Lambda\right)$. The diagram

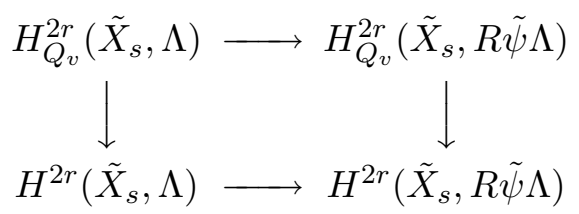

commutes and the lower horizontal map is identified with the specialization map

$$
s p^{*}: H^{2 r}\left(\tilde{X}_{s}, \Lambda\right) \longrightarrow H^{2 r}\left(X_{\bar{t}}, \Lambda\right)
$$


The cycle $\Delta_{v}$ is in the image of $H_{Q_{v}}^{2 r}\left(\tilde{X}_{s}, \Lambda\right)$ in $H^{2 r}\left(X_{\bar{t}}, \Lambda\right)$. Thus, $s p^{*}\left(\Delta_{v}\right)=$ $a \delta_{v}$ with $a \in \Lambda$. Since $\Delta_{v}{ }^{2}=(-1)^{r} 2+0 \in H^{4 r}(Q, \Lambda) \oplus H^{4 r}\left(\hat{X}_{s}, \Lambda\right)=$ $H^{4 r}\left(\tilde{X}_{s}, \Lambda\right)$ and the specialization map is a ring homomorphism, we have

$$
(-1)^{r} 2=\left\langle s p^{*}\left(\Delta_{v}{ }^{2}\right)\right\rangle=\left\langle s p^{*}\left(\Delta_{v}\right)^{2}\right\rangle=\left\langle a^{2} \delta_{v}{ }^{2}\right\rangle=(-1)^{r} 2 a^{2},
$$

hence $a= \pm 1$, or $s p^{*}\left(\Delta_{v}\right)= \pm \delta_{v}$. Since the vanishing cycle $\delta_{v}$ (and $\Delta_{v}$ ) are defined up to sign, we see the image of $\Delta_{v}$ is the vanishing cycle $\delta_{v}$.

2.6. Now we return to the proof of Lemma 2.4. Assume that $0 \leq p<$ $2 r$. Notice that the pull-back

$$
H^{2 p}\left(X_{s}, \Lambda\right) \longrightarrow H^{2 p}\left(\tilde{X}_{s}, \Lambda\right)
$$

is injective since the diagram

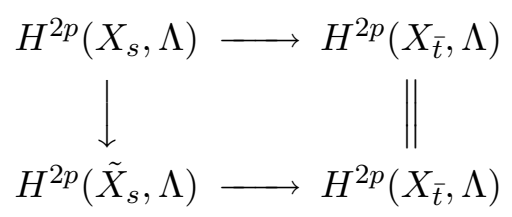

commutes and the upper horizontal map is injective. We have also the commutative diagram

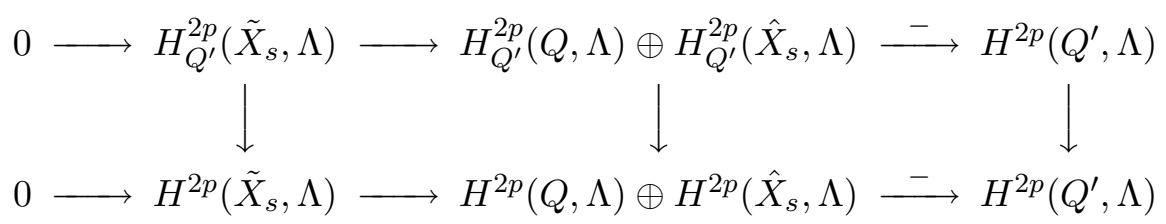

and the middle vertical map is injective. In particular, the left vertical map is also injective. It also follows that $H^{2 p-2}\left(Q^{\prime}, \Lambda\right)=H_{Q^{\prime}}^{2 p}\left(\tilde{X}_{s}, \Lambda\right)$ in $H^{2 p}\left(\tilde{X}_{s}, \Lambda\right)$. The composite of the lower left horizontal map and the pullback $H^{2 p}\left(X_{s}, \Lambda\right) \rightarrow H^{2 p}\left(\tilde{X}_{s}, \Lambda\right)$ is of the form (0, pull-back). Since the upper left horizontal map is "diagonal", i.e., it is identified with the diagonal map $\Lambda \rightarrow \Lambda \oplus \Lambda$ for $0 \leq p \leq 2 r-1, H^{2 p}\left(X_{s}, \Lambda\right)$ injects into the cokernel of 
$H_{Q^{\prime}}^{2 p}\left(\tilde{X}_{s}, \Lambda\right) \rightarrow H^{2 p}\left(\tilde{X}_{s}, \Lambda\right)$. Consider the commutative diagram

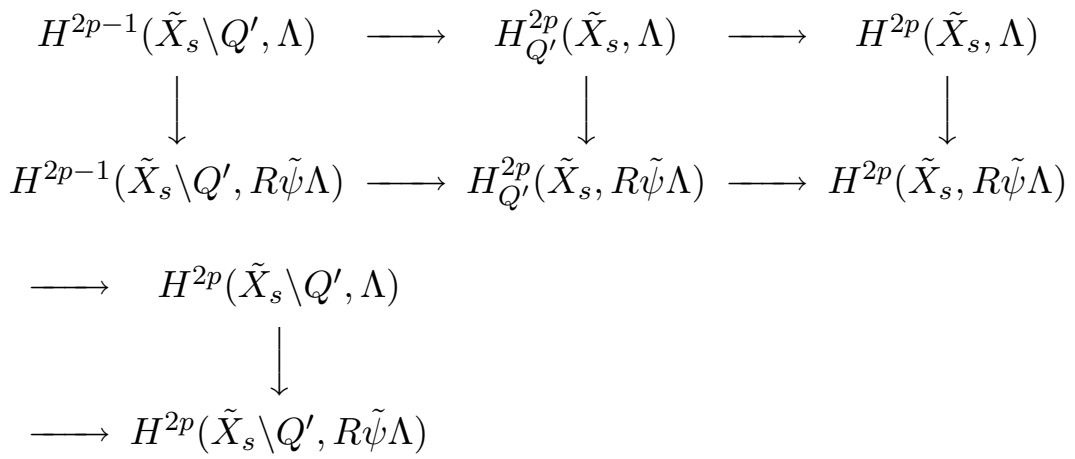

where the horizontal rows are exact. Since $\tilde{X} \rightarrow S$ is smooth on $\tilde{X}_{s} \backslash Q^{\prime}$, the vanishing functor vanishes on $\tilde{X}_{s} \backslash Q^{\prime}$, and hence $\Lambda \cong R \tilde{\psi} \Lambda$ there. It follows that the both vertical extreme arrows are bijective. The injectivity of the upper second map gives a commutative diagram

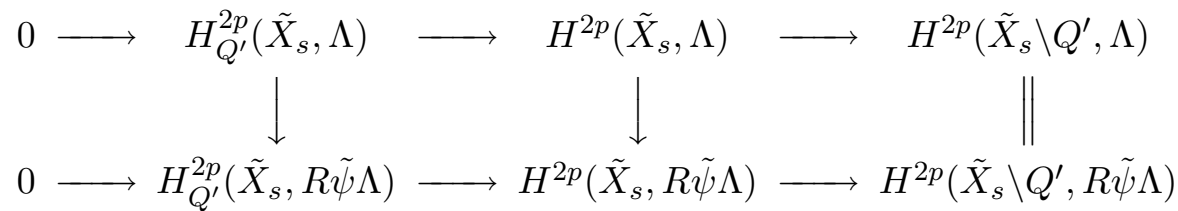

with exact rows. As $H^{2 p}\left(X_{s}, \Lambda\right)$ injects into the cokernel of $H_{Q^{\prime}}^{2 p}\left(\tilde{X}_{s}, \Lambda\right) \rightarrow$ $H^{2 p}\left(\tilde{X}_{s}, \Lambda\right)$, the maps

$$
\begin{gathered}
H^{2 p}\left(X_{s}, \Lambda\right) \longrightarrow H^{2 p}\left(\tilde{X}_{s}, \Lambda\right) \longrightarrow H^{2 p}\left(\tilde{X}_{s} \backslash Q^{\prime}, \Lambda\right) \\
H^{2 p}\left(X_{s}, \Lambda\right) \longrightarrow H^{2 p}\left(\tilde{X}_{s}, \Lambda\right) \longrightarrow H^{2 p}\left(\tilde{X}_{s}, R \tilde{\psi} \Lambda\right)=H^{2 p}\left(X_{\bar{t}}, \Lambda\right)
\end{gathered}
$$

are injective. Moreover, the latter map is bijective for $p \neq r$, and otherwise

$$
0 \longrightarrow H^{2 r}\left(X_{s}, \Lambda\right) \longrightarrow H^{2 r}\left(X_{\bar{t}}, \Lambda\right) \stackrel{\left(\delta_{v}, ?\right)}{\longrightarrow} \bigoplus_{v} \Lambda v
$$

is exact. Therefore, in view of 2.5 in the case of $p=r$, the maps $s p^{*}: H^{2 p}\left(\tilde{X}_{s}, \Lambda\right) \rightarrow H^{2 p}\left(X_{\bar{t}}, \Lambda\right)$ are surjective when tensored with $\mathbf{Q}$ and the torsion elements of $H^{2 p}\left(X_{\bar{t}}, \Lambda\right)$ come from $H^{2 p}\left(X_{s}, \Lambda\right)$. The image of $H_{Q^{\prime}}^{2 p}\left(\tilde{X}_{s}, \Lambda\right) \cong H^{2 p-2}\left(Q^{\prime}, \Lambda\right)$ in $H^{2 p}\left(X_{\bar{t}}, \Lambda\right)$ is torsion, and it comes from $H^{2 p}\left(X_{s}, \Lambda\right)$. Since its image in $H^{2 p}\left(\tilde{X}_{s} \backslash Q^{\prime}, \Lambda\right) \cong H^{2 p}\left(\tilde{X}_{s} \backslash Q^{\prime}, R \tilde{\psi} \Lambda\right)$ vanishes, the image of $H_{Q^{\prime}}^{2 p}\left(\tilde{X}_{s}, \Lambda\right)$ in $H^{2 p}\left(X_{\bar{t}}, \Lambda\right)$ must vanish. Hence, the images 
of $C H_{2 r-p}\left(Q^{\prime}\right) \hookrightarrow C H^{p}\left(\tilde{X}_{s}\right)$ in $H^{2 p}\left(X_{\bar{t}}, \Lambda\right)$ vanish for $0 \leq p \leq 2 r$. (The case $p=2 r$ is trivial.) Since the composition $C H^{p}\left(\tilde{X}_{s}\right) \rightarrow H^{2 p}\left(\tilde{X}_{s}\right) \stackrel{s p^{*}}{\longrightarrow} H^{2 p}\left(X_{\bar{t}}\right)$ is a ring homomorphism, $C H^{\cdot+1}\left(X_{s} \rightarrow X\right) \rightarrow H^{2 \cdot}\left(X_{\bar{t}}\right)$ is also a ring homomorphism. Thus, Lemma 2.4 is proven.

2.7. The theorem follows from the lemma by the functoriality except the last part. It remains to show that the composite $C H^{p}\left(X_{\bar{t}}\right) \rightarrow$ $C H^{p+1}\left(X_{s} \rightarrow X\right) \rightarrow H^{2 p}\left(X_{\bar{t}}\right)$ is in fact the cycle map. By definition, the map is given as follows. Let $s \rightarrow S^{\prime}$ be a pointed open smooth curve, smooth over $S$ outside $s$. The map $C H^{p}\left(\tilde{X}_{S^{\prime}}\right) \rightarrow C H^{p}\left(\tilde{X}_{S^{\prime}} \backslash \tilde{X}_{s}\right)$ is surjective and induces the map $C H^{p}\left(\tilde{X}_{S^{\prime}} \backslash \tilde{X}_{s}\right) \rightarrow C H^{p}\left(\tilde{X}_{s}\right)$ by restriction. We have the cycle map $C H^{p}\left(\tilde{X}_{s}\right) \rightarrow H^{2 p}\left(\tilde{X}_{s}\right)$ and the specialization map $H^{2 p}\left(\tilde{X}_{s}\right) \rightarrow H^{2 p}\left(X_{\bar{t}}\right)$, hence we get $C H^{p}\left(\tilde{X}_{s}\right) \rightarrow H^{2 p}\left(\tilde{X}_{s}\right)$ and the map $C H^{p}\left(\tilde{X}_{S^{\prime}} \backslash \tilde{X}_{s}\right) \rightarrow H^{2 p}\left(X_{\bar{t}}\right)$. The inductive limit of these maps for various $S^{\prime}$ gives the desired map. Thus it suffices to show that for $c \in C H^{p}\left(\tilde{X}_{S^{\prime}}\right)$, the image of the pull-back $\left.c\right|_{\tilde{X}_{s}}$ by the map $C H^{p}\left(\tilde{X}_{s}\right) \rightarrow H^{2 p}\left(\tilde{X}_{s}\right) \rightarrow H^{2 p}\left(X_{\bar{t}}\right)$ is the class of $\left.c\right|_{X_{\bar{t}}}$ in $H^{2 p}\left(X_{\bar{t}}\right)$. If $b: Y \rightarrow \tilde{X}_{S^{\prime}}$ is a resolution of singularities and if $c^{\prime}=b^{*}(c) \in C H^{p}(Y)$, then the class of $\left.c\right|_{X_{\bar{t}}}$ in $H^{2 p}\left(X_{\bar{t}}\right)$ is the image of the class of $c^{\prime}$ by the map $H^{2 p}(Y) \rightarrow H^{2 p}\left(X_{\bar{t}}\right)$. Since the map factors as $H^{2 p}(Y) \rightarrow H^{2 p}\left(Y_{s}\right) \rightarrow H^{2 p}\left(X_{\bar{t}}\right)$, where the latter map is the specialization map for $Y \rightarrow X_{S^{\prime}}$, it is sufficient to verify that the image of the class of $c^{\prime}$ by the map $H^{2 p}(Y) \rightarrow H^{2 p}\left(Y_{s}\right)$ is the image of the class of $\left.c\right|_{\tilde{X}_{s}}$ by the map $b_{s}{ }^{*}: H^{2 p}\left(\tilde{X}_{s}\right) \rightarrow H^{2 p}\left(Y_{s}\right)$. The map $S^{\prime} \rightarrow S$ factors as $S^{\prime} \rightarrow S^{\prime \prime} \rightarrow S$, where $S^{\prime \prime} \rightarrow S$ is étale at the image of $s \in S^{\prime}$, and $S^{\prime} \rightarrow S$ of the form $x^{e}=y$, where $x$ and $y$ are the local parameters at the images of the point $s$. Localizing if necessary, we may assume $S^{\prime \prime} \rightarrow S$ is étale. Since the base-change $\psi: X_{S^{\prime \prime}} \rightarrow X$ is étale, the map ? $[\psi]: C H^{p}\left(X_{s} \rightarrow X_{S^{\prime \prime}}\right) \rightarrow C H^{p}\left(X_{s} \rightarrow X\right)$ is bijective. Thus, we may assume $S^{\prime \prime}=S$. The scheme $\tilde{X}_{S^{\prime}}$ has singularities along $Q^{\prime}$ and at each point of $Q^{\prime}$, it is étale-locally isomorphic to $Q^{\prime} \times\left\{(u, v, w) \in \mathbf{A}^{3} ; u v=w^{2 e}\right\}$, which is essentially a surface singularity of type $A_{2 e-1}$. Hence the exceptional divisor of a resolution $Y$ of singularities consists of divisors $E_{1}, \ldots, E_{2 e-1}$, among which each $E_{i}$ has a map $E_{i} \rightarrow Q^{\prime}$ with fibre $\mathbf{P}^{1} ; E_{i}$ and $E_{i+1}$ intersect along a section $Q_{i}^{\prime}$; and $E_{i}$ and $E_{j}$ do not intersect for $|i-j|>1$. Moreover, $\hat{X}_{s}$ intersects with $E_{1}$ along $Q_{0}^{\prime}$ which is isomorphic to $Q^{\prime}$ and with no other exceptional divisors. Similarly, $Q$ intersects with $E_{2 e-1}$ along $Q_{2 e-1}^{\prime}$ which is isomorphic to $Q^{\prime}$ and with no other exceptional divisors. Denoting $E_{0}=\hat{X}_{s}$ and $E_{2 e}=Q$, we have $Y_{s}=\bigcup_{i=0}^{2 e} E_{i}$. Put $Y^{\prime}=\coprod_{i=0}^{2 e} E_{i}$. Then the natural map $Y^{\prime} \rightarrow Y_{s}$ gives an 
injection

$$
0 \longrightarrow H^{2 p}\left(Y_{s}\right) \longrightarrow H^{2 p}\left(Y^{\prime}\right)
$$

Put $X^{\prime}=E_{0} \coprod E_{2 e}$. Thus we get the commutative diagram

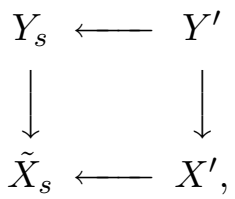

where the right vertical arrow is the identity on $E_{0}$ and $E_{2 e}$; and is, on the other $E_{i}$ 's, the projection $E_{i} \rightarrow Q^{\prime}=Q_{0}^{\prime} \subset E_{0}$. We get a commutative diagram

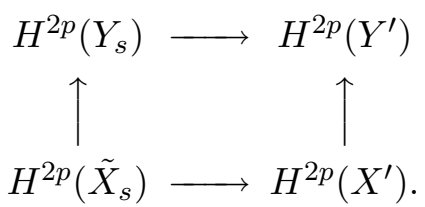

Since $X^{\prime}$ and $Y^{\prime}$ are smooth, we have the commutative diagram

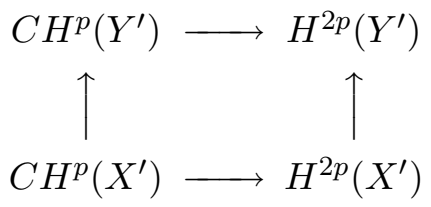

where the horizontal maps are the cycle maps. Similarly, the diagram

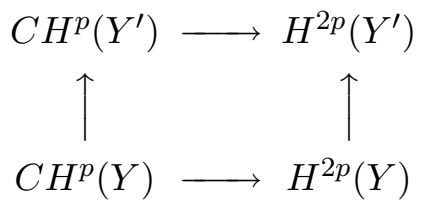

is also commutative. Combining these, we see that the image of the class of $c^{\prime}$ by the map $H^{2 p}(Y) \rightarrow H^{2 p}\left(Y_{s}\right)$ is the image of the class of $\left.c\right|_{\tilde{X}_{s}}$ by the $\operatorname{map} b_{s}{ }^{*}: H^{2 p}\left(\tilde{X}_{s}\right) \rightarrow H^{2 p}\left(Y_{s}\right)$.

\section{REFERENCES}

[B] N. Bourbaki, Éléments de mathématique, Algèbre commutative, Hermann, 1967. [SGA 7] P. Deligne and N. Katz, Groupes de monodromie en géométrie algébrique II, Lecture notes in mathematics, vol. 340, Springer-Verlag, Berlin-Heidelberg-New York, 1973. 
[F] W. Fulton, Intersection theory, Springer-Verlag, 1984.

[EGA] A. Grothendieck and J. Dieudonne, Éléments de géométrie algébrique, Publications mathématiques de IHES, No. 4, 8, 11, 17, 20, 24, 28, 32.

[H] W. V. D. Hodge and D. Pedoe, Methods of algebraic geometry, volume 2, Cambridge University Press, 1952.

[K] Shun-ichi Kimura, Fractional intersection and bivariant theory, Comm. Algebra, 20 (1992), no. 1, 285-302.

Department of mathematics

Nagoya University

Furocho, Chikusa-ku, Nagoya

Japan

saito@math.nagoya-u.ac.jp 